\title{
Targeted Metabolomics with Quantitative Dissolution Dynamic Nuclear Polarization
}

\author{
Lerche, Mathilde Hauge; Karlsson, Magnus; Ardenkjær-Larsen, Jan Henrik; Jensen, Pernille Rose
}

Published in:

NMR-Based Metabolomics

Link to article, DOI:

10.1007/978-1-4939-9690-2_21

Publication date:

2019

Document Version

Peer reviewed version

Link back to DTU Orbit

Citation (APA):

Lerche, M. H., Karlsson, M., Ardenkjær-Larsen, J. H., \& Jensen, P. R. (2019). Targeted Metabolomics with Quantitative Dissolution Dynamic Nuclear Polarization. In G. Gowda, \& D. Raftery (Eds.), NMR-Based Metabolomics (pp. 385-393). Springer. Methods in Molecular Biology Vol. 2037 https://doi.org/10.1007/978-14939-9690-2_21

\section{General rights}

Copyright and moral rights for the publications made accessible in the public portal are retained by the authors and/or other copyright owners and it is a condition of accessing publications that users recognise and abide by the legal requirements associated with these rights.

- Users may download and print one copy of any publication from the public portal for the purpose of private study or research.

- You may not further distribute the material or use it for any profit-making activity or commercial gain

- You may freely distribute the URL identifying the publication in the public portal 


\title{
Targeted Metabolomics with Quantitative Dissolution Dynamic Nuclear Polarization
}

\author{
Mathilde H. Lerche, Magnus Karlsson, Jan H. Ardenkjær-Larsen, \\ and Pernille R. Jensen
}

\begin{abstract}
Metabolite profiles and their isotopomer distributions can be studied noninvasively in complex mixtures with NMR. The advent of hyperpolarized ${ }^{13} \mathrm{C}-\mathrm{NMR}$ using quantitative dissolution Dynamic Nuclear Polarization (qdDNP) and isotope enrichment add sensitivity to such metabolic studies, enabling mapping and quantification of metabolic pathways and networks. Here we describe a sample preparation method, including cell incubation, extraction, and signal enhancement, for reproducible and quantitative analysis of hyperpolarized ${ }^{13} \mathrm{C}-\mathrm{NMR}$ metabolite spectra. We further illustrate how qdDNP can be applied to gain metabolic insights into living cells.
\end{abstract}

Key words dDNP, Hyperpolarization, ${ }^{13} \mathrm{C}-\mathrm{NMR}$, Glucose

\section{Introduction}

Targeted metabolomics or stable isotope resolved metabolomics (SIRM) measures an isotope-filtered selection of molecules aimed at understanding the dynamics and compartmentation of metabolic pathways and networks $[1,2]$. The metabolic transformations of one or more isotope-labeled tracers lead to distinct labeling patterns in metabolic intermediates. Using such patterns, metabolic fluxes can be measured from temporal profiles of metabolite concentration changes. A widely used isotope-labeled tracer is $\left[\mathrm{U}_{-}{ }^{13} \mathrm{C}\right]$ glucose, which provides valuable insight into the glycolytic pathway, the pentose phosphate pathway, labeling of intermediates in the TCA cycle, and labeling of amino acids. In conjunction with the use of stable isotope tracers, ${ }^{13} \mathrm{C}-\mathrm{NMR}$ is a method of choice for exploring metabolic reprogramming in major metabolic diseases such as cancer and diabetes $[3,4] .{ }^{13} \mathrm{C}-\mathrm{NMR}$ has a number of unique advantages for metabolic studies; it is quantitative, versatile, and specific. The relative insensitivity of the method can be 
compensated when combined with ${ }^{13} \mathrm{C}$ hyperpolarization in the form of dissolution dynamic nuclear polarization [dDNP] [5]. DNP make use of the transfer of electron spin polarization from a free radical to, e.g., ${ }^{13} \mathrm{C}$ nuclear spins mediated by resonant microwave irradiation [6]. This transfer is best conducted in amorphous samples that assure the homogenous distribution of electrons and nuclear spins. DNP is typically performed at low temperatures $(<1.5 \mathrm{~K})$ and at high magnetic fields $(>3 \mathrm{~T})$ where the electron spin polarization approaches 100\%. Following completed hyperpolarization, hot solvent dissolves the sample, and the sample is transferred to a room temperature NMR magnet for analysis. Whereas hyperpolarization losses during this dissolution step can be kept to a minimum, a principal limitation for analyzing hyperpolarized molecules is short signal lifetime of seconds to a few minutes for non-protonated ${ }^{13} \mathrm{C}$ sites in small molecules in the liquid state, which is governed by the nuclear $T_{1}$ relaxation time. Here we demonstrate how to combine targeted metabolomics methodology with hyperpolarized ${ }^{13} \mathrm{C}-\mathrm{NMR}$ (Fig. 1). We further show how the method can be developed to make absolute quantifications of metabolic fluxes [7] (Fig. 2). The method is illustrated in a longitudinal study on how $\beta$-cells handle increased fuel pressure (Fig. 3).
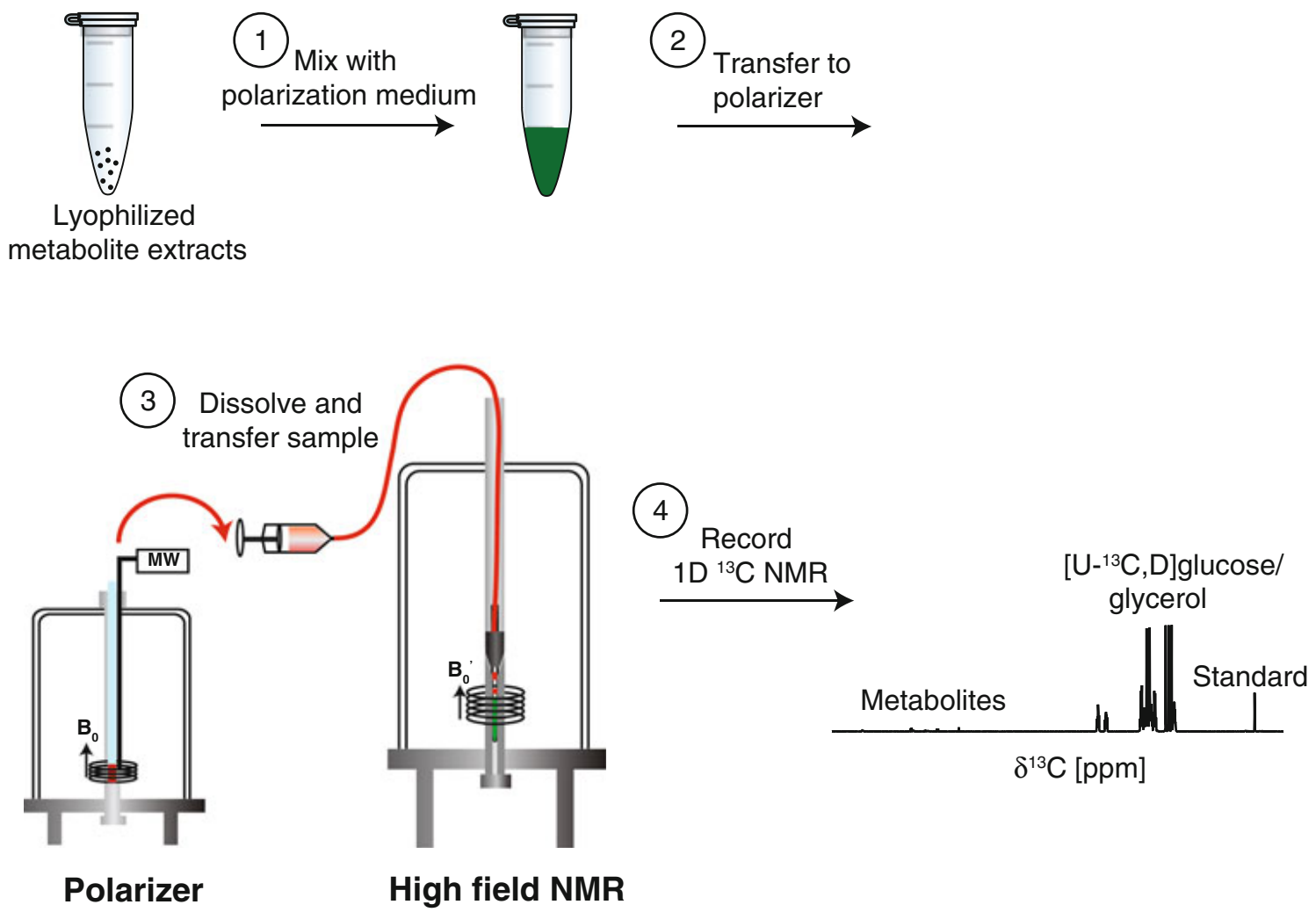

Fig. 1 Principle steps in the qdDNP-NMR analysis method. A description of the four steps in the analysis is found in Subheading 3.2 


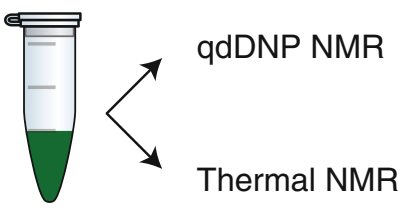

\begin{tabular}{|l|c|c|c|}
\hline $\begin{array}{c}\text { Compound } \\
(\mathrm{Com})\end{array}$ & $\begin{array}{c}\text { DNP ratio } \\
(\mathrm{Com} / \mathrm{HP01})\end{array}$ & $\begin{array}{c}\text { NMR ratio } \\
(\mathrm{Com} / \mathrm{HP01})\end{array}$ & SLC \\
\hline Lactate-C1 & $0.55 \pm 0.03$ & $0.67 \pm 0.07$ & $82 \pm 8 \%$ \\
\hline Glutamate-C5 & $0.66 \pm 0.03$ & $1.34 \pm 0.22$ & $49 \pm 9 \%$ \\
\hline Alanine-C1 & $0.60 \pm 0.04$ & $1.08 \pm 0.01$ & $55 \pm 4 \%$ \\
\hline Pyruvate-C1 & $0.57 \pm 0.09$ & $0.53 \pm 0.01$ & $108 \pm 9 \%$ \\
\hline
\end{tabular}

Fig. 2 Calculation method for signal loss coefficient (SLC)

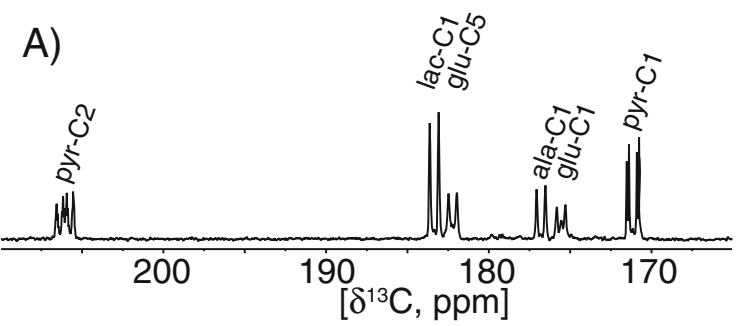

B)

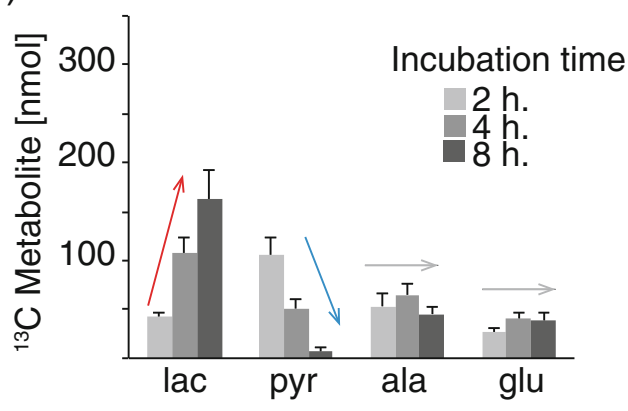

Fig. 3 Illustration of metabolite quantification in qdDNP-NMR experiments. (a) Typical ${ }^{13} \mathrm{C}$ NMR spectrum from the metabolite extract of $\beta$-cells incubated with $11.7 \mathrm{mM}\left[\mathrm{U}-{ }^{13} \mathrm{C}, \mathrm{D}\right] \mathrm{glucose}$ for $4 \mathrm{~h}$. (b) Quantification of glucose-derived metabolites (lactate, pyruvate, alanine, and glutamate) in $\beta$-cells in a longitudinal study $(2,4$, and $8 \mathrm{~h}, 11.7 \mathrm{mM})$. Constant production of lactate (red arrow, $0.65 \mathrm{nmol} / \mathrm{min}$ ), depletion of pyruvate (blue arrow, $-0.3 \mathrm{nmol} / \mathrm{min}$ ), and constant pool of glutamate (TCA cycle) and alanine (grey arrows) are determined 


\section{Materials}

2.1 Stable IsotopeLabeled Cell Culture Extract Samples and Reference Compounds

\subsubsection{Chemicals}

\subsubsection{Cell Extract} Samples

\subsubsection{Reference Samples}

\subsection{DNP \\ Hyperpolarization}

$2.3{ }^{13} \mathrm{C}-\mathrm{NMR}$

2.4 Example: $\beta$-Cell Metabolism
1. HP001 (1,1-bis (hydroxymethyl- $\mathrm{D}_{2}$ ) cyclopropane-1-13C, $\mathrm{D}_{4}$ ).

2. OX063 (trityl radical).

3. ProHance ${ }^{\mathrm{TM}}$ (MR contrast agent).

4. OmniScan ${ }^{\mathrm{TM}}$ (MR contrast agent).

1. Isotope-labeled substrate: stock solution of $120 \mathrm{mM}\left[\mathrm{U}^{-13} \mathrm{C}\right.$, $\mathrm{D}_{7}$ ]glucose (Sigma-Aldrich) dissolved in desired incubation medium.

2. Polarization medium: $70 \mathrm{mg}$ OX063 (electron polarizer agent, EPA), $1227 \mathrm{mg}$ glycerol (GS), $944 \mathrm{mg}$ Milli-Q water, and $6 \mu \mathrm{L}$ ProHance $^{\mathrm{TM}}(0.5 \mathrm{M}$, electron relaxation agent, ERA).

1. DNP sample composition.

Stock solution is made using $0.75 \mu \mathrm{mol}{ }^{13} \mathrm{C}$ of each desired compound (corresponding to $75 \mu \mathrm{mol}$ of natural abundance chemicals) mixed with $450 \mu \mathrm{L}$ polarization medium, $0.75 \mu \mathrm{mol}$ $\left[1-{ }^{13} \mathrm{C}\right] \mathrm{HP00l}(15 \mu \mathrm{L}, 50 \mathrm{mM})$, and $5.4 \mathrm{mg}\left[\mathrm{U}_{-}{ }^{13} \mathrm{C}, \mathrm{D}\right]$ glucose. A $75 \mu \mathrm{L}$ aliquot of DNP sample composition is hyperpolarized and dissolved as described in the methods $(n=3)$.

2. Thermal NMR sample composition.

A $75 \mu \mathrm{L}$ aliquot of DNP sample composition is dissolved in $400 \mu \mathrm{L}$ phosphate buffer $(40 \mathrm{mM}, \mathrm{pH} 7.3), 75 \mu \mathrm{L} \mathrm{D}_{2} \mathrm{O}$, and $15 \mu \mathrm{L}$ OmniScan $(n=3)$.

Dynamic nuclear polarization was performed in a HyperSense (Oxford Instruments) polarizer with microwave irradiation at a frequency of $94 \mathrm{GHz}$ and a power of $100 \mathrm{~mW}$. All samples were polarized for $90 \mathrm{~min}$. The microwave frequency and power should be optimized for the specific polarizer instrument according to established procedures.

Hyperpolarized $1 \mathrm{D}{ }^{13} \mathrm{C}$ NMR spectra were recorded on a $9.4 \mathrm{~T}$ NMR Varian spectrometer. Thermal $1 \mathrm{D}{ }^{13} \mathrm{C}$ NMR spectra were acquired on a 19 T NMR Bruker spectrometer equipped with a cryoprobe.

Cell culture: INS-1 cells were cultured in Gibco RPMI 1640 Medium, GlutaMAX Supplement (Thermo Fisher Scientific) with $10 \%$ fetal bovine serum (FBS) (Sigma-Aldrich), 1\% PenicillinStreptomycin (Sigma-Aldrich), and $500 \mu \mathrm{L}$ of $50 \mathrm{mM}$ 2-mercaptoethanol (Thermo Fisher Scientific). This culture medium contains $11 \mathrm{mM}$ glucose. The culture was maintained at 
$37{ }^{\circ} \mathrm{C}$ under humidified ( $5 \% \mathrm{CO}_{2}, 95 \%$ air) conditions. Cell number and viability were measured by an automatic cell counter (EVE, NanoEntek). Cells were sub-cultured in $175 \mathrm{~cm}^{2}$ flasks at a density of $3 \times 10^{6}$ cells for 7 days.

\section{Methods}

\subsection{Metabolite Component Sample (see Note 1)}

\subsection{Dissolution Dynamic Nuclear Polarization}

1. Grow human cells according to available cell-specific protocols (e.g., ATCC). Harvest cells by trypsinization. Wash and redissolve cells in desired medium (e.g., cell culture medium without glucose, PBS, or hypotonic phosphate buffer) to a concentration of $20 \times 10^{6}$ cells $/ \mathrm{mL}$ ( see Note 2$)$.

2. Incubate $500 \mu \mathrm{L}$ cells in suspension in $2 \mathrm{~mL}$ Eppendorf tubes with $100 \mu \mathrm{L}, 120 \mathrm{mM}$ uniformly ${ }^{13} \mathrm{C}$-isotope-labeled and partially deuterated glucose in a shaking thermostat $\left(37^{\circ} \mathrm{C}\right.$ and $500 \mathrm{rpm}$ ) for $30 \mathrm{~min}$ or longer (see Note 3 ).

3. Stop all enzymatic reactions immediately by addition of $400 \mu \mathrm{L}$ ice-cold 2.2 M perchloric acid (PCA) to the $600 \mu \mathrm{L}$ substrate incubated cell suspension and place on ice for at least $10 \mathrm{~min}$. Extract the water soluble metabolites by centrifugation $\left(10 \mathrm{~min}, 9600 \times \mathrm{g}, 4^{\circ} \mathrm{C}\right)$. Neutralize the supernatant with $3 \mathrm{M} \mathrm{KOH}$ (add pH indicator for visual inspection of neutralization). In order to obtain a high recovery of metabolites, it is important not to over titrate the samples and keep them on ice to prevent heating. Leave the neutralized sample on ice for at least $10 \mathrm{~min}$. Remove the precipitated salt by centrifugation $\left.(10 \mathrm{~min}, 9600 \times g), 4^{\circ} \mathrm{C}\right)($ see Note 4$)$.

4. Lyophilize the extract ( see Note 5).

1. To hyperpolarize the freeze-dried extract efficiently, a glassing solvent (GS), an electron polarization agent (EPA, source of free radicals) and an electron relaxation agent (ERA, paramagnetic compound) are needed (together referred to as the polarization medium) [8]. Each lyophilized extract contained in an Eppendorf tube is dissolved in a total of $150 \mu \mathrm{L}$ of polarization medium. To each sample, an internal standard $(5 \mu \mathrm{L}$ of a $50 \mathrm{mM}$ water solution of HP001) is added. The sample is whirl-mixed and sonicated for a few minutes to ensure that the entire extract is dissolved and subsequently centrifuged (see Note 6).

2. The sample is pipetted into a sample holder and inserted into the polarizer. Microwaves are activated. The samples are fully polarized after $90 \mathrm{~min}$. Since the metabolite quantification is performed relative to the added standard, it is possible to harvest the polarized samples earlier (down to $30 \mathrm{~min}$ polarization time) with the penalty of a lower signal-to-noise ratio. 


\subsection{Absolute} Quantification
3. After completing the hyperpolarization, the samples are dissolved in $5 \mathrm{~mL}$ of $40 \mathrm{mM}$ phosphate buffer ( $\mathrm{pH} \mathrm{7.3).} \mathrm{The}$ sample is transferred to a $5 \mathrm{~mm}$ NMR tube and inserted in a NMR magnet tuned to ${ }^{13} \mathrm{C}$ for analysis (see Note 7 ). The presented data are based on a total sample transfer time of 10-12 s and a sample temperature starting at $50{ }^{\circ} \mathrm{C}$ immediately after dissolution and ending at $38 \pm 2{ }^{\circ} \mathrm{C}$ at the time of the NMR acquisition ( see Note 8).

4. A direct detection $1 \mathrm{D}^{13} \mathrm{C}$ NMR spectrum with proton decoupling is recorded with a $90^{\circ}$ pulse, immediately after insertion of the sample into the magnet. Processing and analysis of the spectrum is performed with any available NMR analysis program, e.g., MNOVA [9].

1. For quantification of the metabolites, an internal standard is added. The choice of standard is highly important with several requirements needing to be fulfilled: chemical inertness, a long longitudinal relaxation time $\left(T_{1}\right)$, and a singlet signal not overlapping with a metabolite resonance in NMR spectrum. The ${ }^{13}$ C-labeled HP001 is chosen for these reasons. With this standard, the metabolites in the cellular extract are quantified as a ratio between the integral of an identified metabolite signal relative to the integral of HP001. This ratio is stable and independent of the polarization time for individual metabolites with a coefficient of variation below $15 \%$.

2. For absolute quantification, it is necessary to account for signal decay during the transfer between the polarizer and the NMR magnet for all carbon atoms of interest in the individual metabolites. This can be done by calculating a signal loss coefficient (SLC) for the individual carbon atoms. Samples of isotopelabeled or non-labeled compounds of interest are prepared using the polarization medium described in Subheading 3.2, step 1. For ${ }^{13} \mathrm{C}$-labeled chemicals, several compounds can be analyzed in the same sample by spiking known amounts of compounds into the polarization medium. It is very important to make sure that the compounds are fully dissolved in the polarization medium; sonication and heating may aid the process. The samples are split into two parts where one part is analyzed with hyperpolarized ${ }^{13} \mathrm{C}$ NMR and the other part with thermal ${ }^{13} \mathrm{C}$ NMR. The SLCs are calculated using the respective ratios of ${ }^{13} \mathrm{C}$ NMR signals from a metabolite carbon and the internal standard. The part of the reference sample analyzed with ${ }^{13} \mathrm{C}$ hyperpolarized NMR is hyperpolarized, dissolved, and analyzed as described in Subheading 3.2, steps 2-4. For the other part of the reference sample, a ${ }^{13} \mathrm{C}$ thermal NMR spectrum is recorded on a high-field spectrometer with 4096 scans and a delay of $2 \mathrm{~s}$ between $60^{\circ}$ pulses. Differences in 
sample preparation such as varying concentrations and different labeling schemes do not influence the calculated SLC.

3. A SLC was calculated for the carbonyl atoms of four oftenoccurring metabolites $\left(1-{ }^{13} \mathrm{C}\right.$-lactate, $5-{ }^{13} \mathrm{C}$-glutamate, $1-{ }^{13} \mathrm{C}$ alanine, and $1-{ }^{13} \mathrm{C}$-pyruvate). The ratios between the individual metabolite signals and the internal standard are measured from thermal ${ }^{13} \mathrm{C}$ NMR and hyperpolarized ${ }^{13} \mathrm{C}$ NMR data, respectively. From these ratios, the individual SLC is calculated (Fig. 2) (see Note 9). These SLCs were used to calculate the absolute concentrations of these four metabolites produced by $\beta$-cells exposed to different isotope-labeled glucose loads, Fig. 3 ( see Note 10).

\section{Notes}

1. The qdDNP methodology requires a water-soluble dry extract but does not impose other limitations to the formulated metabolite sample. The metabolite sample preparation could be done in several other ways than described here, e.g., incubation could be done in flasks without trypsinization; separation of intra- and extracellular metabolite fractions could be done; methanol or ethanol extractions could be performed instead of PCA-KOH extraction [10].

2. The stated cell number is a current minimum number of cells used to obtain the final claimed assay sensitivity. The incubation medium is chosen dependent on the study goal. It is important that the medium does not contain the incubation substrate, which is glucose in the described protocol.

3. The cell type (how glycolytic the cells are), cell number, and incubation time all influence the desired substrate concentration. The goal is to apply enough substrate to secure a linear, and small, consumption during the experiment. This requirement can easily be evaluated by changing the substrate concentrations and measuring consumption at different time points and/or cell concentrations using either ${ }^{13} \mathrm{C} \mathrm{NMR}$ or readily available biochemical assays.

4. Not all metabolites are stable in strong acid. If in doubt, then a spiking test is recommended: Add a known concentration of the metabolite in question to the cell sample, extract the metabolite using the PCA- $\mathrm{KOH}$ procedure, freeze-dry the sample and redissolve it in an "NMR buffer," run conventional ${ }^{13} \mathrm{C}-\mathrm{NMR}$, and quantify the metabolite against an added standard.

5. At this point the sample is stable and can be kept frozen until prepared for qdDNP. For this reason, there is no requirement 
for targeted metabolomics with qdDNP to have dDNP equipment in house since the metabolite component samples can be transported to a collaborator laboratory with dDNP equipment available.

6. Trityl radical (OX063) can be purchased from Albeda Research Aps, Denmark. HP001 can be purchased from Sigma-Aldrich on a quote basis (it is not available in their catalogue).

7. Any high-field NMR magnet can be used as long as the signal dispersion is good enough to separate metabolites of interest. A $10 \mathrm{~mm}$ NMR probe can be used advantageously to increase the available signal.

8. This part of the assay is critical. The transfer time, the sample temperature, the sample concentration, and the magnetic environment during the sample transfer all influence the resulting available metabolite signals. These parameters will vary in different hyperpolarization laboratories. The important point is to have the parameters under control such that different samples will undergo the same conditions and be measured relative to a well-characterized standard compound. See Subheading 3.3 on absolute quantitation. If these steps are followed, then only the signal-to-noise ratio will vary between different laboratories.

9. The sensitivity and variation in SLC between different laboratories can be improved significantly. A recent report shows that it may be highly beneficial to perform the DNP at higher field where the polarization can be expected to double [11]. Potentially the largest gain in sensitivity will come from a significant reduction in sample dissolution volume and better utilization of the available sample. A reduction from the current $5 \mathrm{~mL}$ to $1 \mathrm{~mL}$ and the use of a $10 \mathrm{~mm}$ probe instead of a $5 \mathrm{~mm}$ probe can increase the sensitivity 20 times. Implementation of these improvements is important for optimization of assay sensitivity and reproducibility. For absolute quantification, it will always be necessary to have a fully characterized metabolite signal as described above with the calculation of individual carbon SLC. As different hyperpolarization laboratories standardize their protocols, it can be envisioned that the community can produce a common relaxation database similar to the available chemical shift databases-a tool that will significantly improve the described method.

10. The qdDNP-NMR method is valuable in hypothesis-driven research, as shown in the example using $\beta$-cells that can produce easily interpreted quantitative information about cell metabolism. An important perspective of this type of derived metabolic information is its translation to clinical applications where the dDNP MRI methodology is well underway to be implemented as a metabolic contrast agent method useful for diagnosis and therapy monitoring. 


\section{Acknowledgments}

The authors gratefully acknowledge funding by Danish National Research Foundation (grant DNFR124).

\section{References}

1. Nagana Gowda GA, Shanaiah N, Raftery D (2012) Isotope enhanced approaches in metabolomics. Adv Exp Med Biol 992:147-164

2. Bruntz RC, Lane AN, Higashi RM, Fan TWM (2017) Exploring cancer metabolism using stable isotope resolved metabolomics (SIRM). J Biol Chem 292(28):11601-11609

3. Fan TWM, Lorkiewicz PK, Sellers K, Moseley HN, Higashi RM, Lane AN (2012) Stable isotope-resolved metabolomics and applications for drug development. Pharmacol Ther 133(3):366-391

4. Sas KM, Karnovsky A, Michailidis G, Pennathur S (2015) Metabolomics and diabetes: analytical and computational approaches. Diabetes $64(3): 718-732$

5. Ardenkjær-Larsen JH, Frindlund B, Gram A, Hansson G, Hansson L, Lerche MH et al (2003) Increase in signal-to-noise ratio of $>$ 10,000 times in liquid-state NMR. Proc Natl Acad Sci U S A 100(18):10158-10163

6. Abragam A, Goldman M (1978) Principles of dynamic nuclear polarization. Rep Prog Phys 41:395-467
7. Lerche $\mathrm{MH}$, Yigit $\mathrm{D}$, Frahm $\mathrm{AB}$, ArdenkjærLarsen JH, Malinowski RM et al (2018) Stable isotope-resolved analysis with quantitative dissolution dynamic nuclear polarization. Anal Chem 90(1):674-678

8. Karlsson M, Jensen PR, Duus JO, Meier S, Lerche MH (2012) Development of dissolution DNP-MR substrates for metabolic research. Appl Magn Reson 43:223-236

9. Mestrelab Research SL (2015) Mestrenova. https://www.mestrelab.com

10. Sellick CA, Knight D, Croxford AS, Maqsood AR, Stephens GM, Goodacre R et al (2010) Evaluation of extraction processes for intracellular metabolite profiling of mammalian cells: matching extraction approaches to cell type and metabolite targets. Metabolomics $6: 427-438$

11. Capozzi A, Patel S, Ouari O, Karlsson M, Lerche MH, Ardenkjær-larsen JH (2019) Gadolinium effect at High-Magnetic-Field DNP: $70 \%{ }^{13} \mathrm{C}$ Polarization of $\left[\mathrm{U}^{13} \mathrm{C}\right]$ Glucose using Trityl. J Phys Chem Lett vol 10 (12):3420-3425 